\title{
UTILIZACIÓN DEL HÁBITAT POR Salmo trutta $L$.
}

\author{
Rodrigues, L. S. B.*, Cortes, R** y Monzón, A** \\ * Departamento de Engenharia Biológica e Ambiental \\ **Departamento de Florestal. \\ Universidade de Trás-os-Montes e Alto Douro. Apartado 202. 5001 Vila Real Codex; PORTUGAL.
}

Palabras clave: Salmo trutta, Chondrostoma polylepis duriensis, Leuciscus cephalus, macrohábitat, parámetros poblacionales, ordenamiento piscícola.

\begin{abstract}
HABITAT USE BY Salmo trutta L.

It was intented to determine the main physical factors conditioning the use of the rnacrohabitat by $S$. trutta. These ones were the quality of the pool, mean width and depth of the water, macrophyte cover and the amount of fine and coarse particulate organic matter. Fish density (total numbers of the overall species and trout numbers, especially in this case the age classes of $\mathrm{O}+, 1+$ and $2+$ ) could be related to the type of rnacrophyte cover. However, the quality of pool is more important in deterrnining the abundance of the older age classes of this species. On the other hand, fish diversity is mainly linked with the alternance of riffle and pool reaches and their complexity, namely the presence of aquatic macrophytes.
\end{abstract}

\section{INTRODUCCIÓN}

Salmo trutta es un importante recurso nacional e internacional, tanto al nivel de pesca deportiva como comercial. Las poblaciones de esta especie polimórfica, al variar tan considerablemente en su ciclo de vida (ELLIOTT, 1989), son afectadas en sus crecimientos y tasas de supervivencia por numerosos factores, hecho que dificulta la obtención de información científica, esencial para su conservación y gestión.

Los requisitos ambientales de S. trutta han sido prioritariarnente estudiados con vista al correcto ordenamiento piscícola de los ríos y previsión de los impactos futuros en el desarrollo de sus poblaciones. A pesar de los diversos estudios encaminados a establecer criterios objetivos de selección de hábitat, permanece nebuloso, a partir de la literatura existente, si S. trutta tiende a seleccionar hábitats con características similares en diferentes ríos (HEGGENES, 1988b).

\section{ÁREA DE ESTUDIO}

El río Olo nace en la sierra del Alvao, a 1200 m de altitud (región de Trás-os-Montes, Portugal), y recorre una distancia de $39,4 \mathrm{~km}$, en sentido NE-SW, hasta su confluencia con el río Tamega (figura 1). En su perfil, presen- ta pendientes muy acentuadas en los sectores medio y superior.

Este río fue seleccionado por dos razones: por un lado, representa un sistema no eutrofizado que fluye a través de una cuenca de drenaje, con predominio de Quercus pyrenaicus, Pinus pinaster, Erica spp, Ulex spp y Chamaespartium spp, $y$ por otro, es el eje del Parque Natural del Alvao que presenta una creciente presión poblacional.

Este río se caracteriza por presentar aguas rápidas, lecho grosero donde predominan bloques y guijarros, bajo $\mathrm{pH}$ $(\leq 6,5)$ y rnineralización (conductividad $\leq 40.0 \mu \mathrm{mhos} \mathrm{cm}^{-}$), con niveles de oxígeno disuelto elevado (OD $\geq 9,0 \mathrm{mg}^{1-1}$ ). Posee una escasa producción autotrófica y la diversidad de rnacrófitos acuáticos está reducida a Fontinalis antypiretica, Ranunculus peltatus spp peltatus, Callitriche stagnalis y Myosotis sp. Sus márgenes presentan generalmente una densa cobertura riparia de Alnus glutinosa.

\section{MATERIAL Y MÉTODOS}

Atendiendo a los objetivos definidos se estableció una red de diez estaciones de muestreo, en los tramos superior (990 m de altitud) y medio $(240 \mathrm{~m})$ del río Olo, caracterizados en el primer caso por la existencia exclusiva de S. trutta o por constituirse en una zona de transición entre salmónidos y ciprínidos (en el $2^{\circ}$ caso). Para su selección 


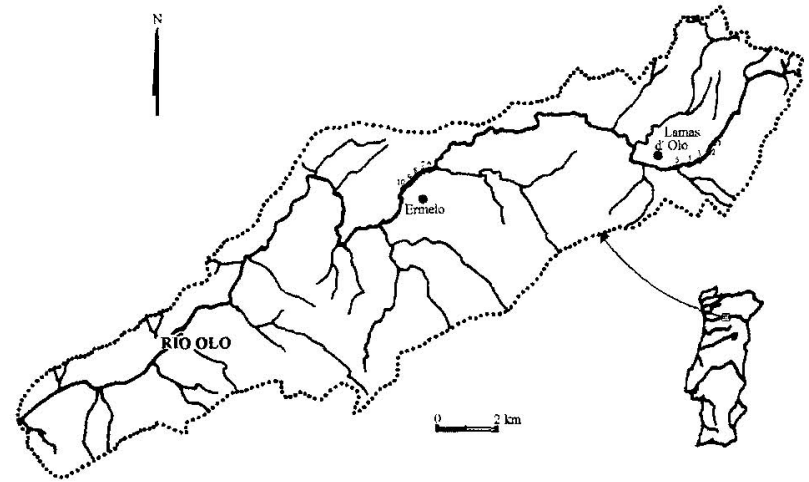

FIGURA 1.- Area de estudio (río Olo) y localización de las estaciones de muestreo.

FIGURE 1.- Study area (River Olo) and location of sampling stations.

se optó como criterio primordial, por la representatividad del trozo estudiado procurando así mismo el máximo de diversidad del hábitat. Las estaciones consideradas presentan una longitud variable, entre 2,5 a $25 \mathrm{~m}$, e intervalos entre sí de 50 a $250 \mathrm{~m}$, aproximadamente.

La población piscícola fue muestreada por pesca eléctrica, usándose un generador portátil Hans Grassl de 1200 w, $500 \mathrm{v}$ e intensidad máxima teórica de $7 \mathrm{~A}$. Redes verticales de malla fueron colocadas en las zonas limítrofes del sector de muestreo. En el terreno, los individuos eran pesados, medida su longitud total y se extraían las escamas para posterior determinación de la edad. Para el retrocálculo de los incrementos de longitud se usó el modelo de Fraser-Lee.

La densidad y biomasa fueron estimadas por el método de Zippin, con dos a cuatro extracciones, dependiendo de la eficacia obtenida. Además, fueron determinados los factores de condición (salmónidos $\left(\mathrm{F}^{\prime}\right)$ y alométrico $\left(\mathrm{K}^{\prime}\right)$ ), la producción a través del modelo de Ricker $(\mathrm{P})$, tasa de renovación (P/B) y los parámetros de crecimiento siguientes: longitud máxima teórica $\left(\mathrm{L}_{\infty}\right)$ del modelo Von Bertalanffy, longitud teórica para $\mathrm{t}=\mathrm{O}\left(\mathrm{L}_{\mathrm{o}}\right)$, tasa específica instantánea de crecimiento (G.g) y la tasa instantánea de decrecimiento de la variable anterior $(\mathrm{g})$, todos ellos obtenidos mediante el método de Gompertz.

En la estima del uso del macrohábitat por S. trutta se midieron las variables ambientales que caracterizan las secciones en estudio, utilizándose algunos de los criterios propuestos por PLATTS et al. $(1983,1987)$. Se establecieron de dos a tres transectos en cada estación, de acuerdo con la heterogeneidad del medio físico, determinándose cada $20 \mathrm{~cm}$ los siguientes parámetros: profundidad de la columna de agua $(\mathrm{cm})(\mathrm{PM}), \%$ de cobertura de gramíneas
(GC), \% de cobertura de macrófitos (CM), \% de materia orgánica particulada fina (FPOM) y gruesa (CPOM), proporción del sustrato dominante y subdominante (\%) (SD) empleándose la escala de Wentworth, \% de la tasa de material embebido (EM), razón léntica-lótica (PR) y velocidad de corriente $\left(\mathrm{cm} \mathrm{s}^{-1}\right)$ (VC).

En cada una de las estaciones se determinaron los siguientes parámetros: anchura media (LM), profundidad media (PM), gradiente del canal (GC), \% de sombra ripícola (ES) y calidad de la zona léntica (QP). Todos los datos obtenidos en porcentajes son transformados por arcsen $\sqrt{x}$ (SOKAL \& ROHLF, 1969).

Para determinar la influencia de los parámetros fisicoquímicos y ambientales sobre los diversos parámetros de la estructura poblacional de S. trutta, se aplicó una regresión múltiple paso a paso y correlación de Pearson. El análisis canónico de correspondencias (CCA) (TER BRAAK, 1987), fue utilizado para relacionar las variables del hábitat con las densidades de $\mathrm{S}$ trutta así como con la de las otras especies de ciprínidos que cohabiten simultáneamente con ésta. Para ese fin se utilizaron matrices de densidades piscícolas y matrices de variables ambientales. Estas ultimas recogen únicamente aquellas variables cuyas correlaciones de Pearson con variables biológicas presenten los valores más elevados, independientemente de si son o no significativos $(\mathrm{p} \leq 0,05)$. Todos los datos fueron transformados por $\log (x+1)$.

\section{RESULTADOS}

En la región de transición entre salmónidos y ciprínidos (sector B) se verifica que S. trutta presenta un papel relevante en la comunidad, principalmente en los sectores superiores (figura 2). La estructura de edades de S. trutta presenta diferencias sustanciosas en las diferentes regiones definidas (figura 3 ):

- En el sector de salmónidos (sector A) la edad máxima observada fue de 4+, encontrándose en contrapartida edades $5+$ y $6+$ en el tramo inferior siguiente (sector B).

- En la región de transición, las poblaciones de individuos de edad $1+$, presentan cuantitativos extremadamente bajos.

A través de la correlación de Pearson (tabla 1) se verifica que:

- La longitud máxima teórica del modelo de Gompetz para S. trutta se encuentra positivamente correlacionada con la anchura media del río (LM) y con la variedad en materia orgánica particulada (DSFPOM, CPOM y DSC- 

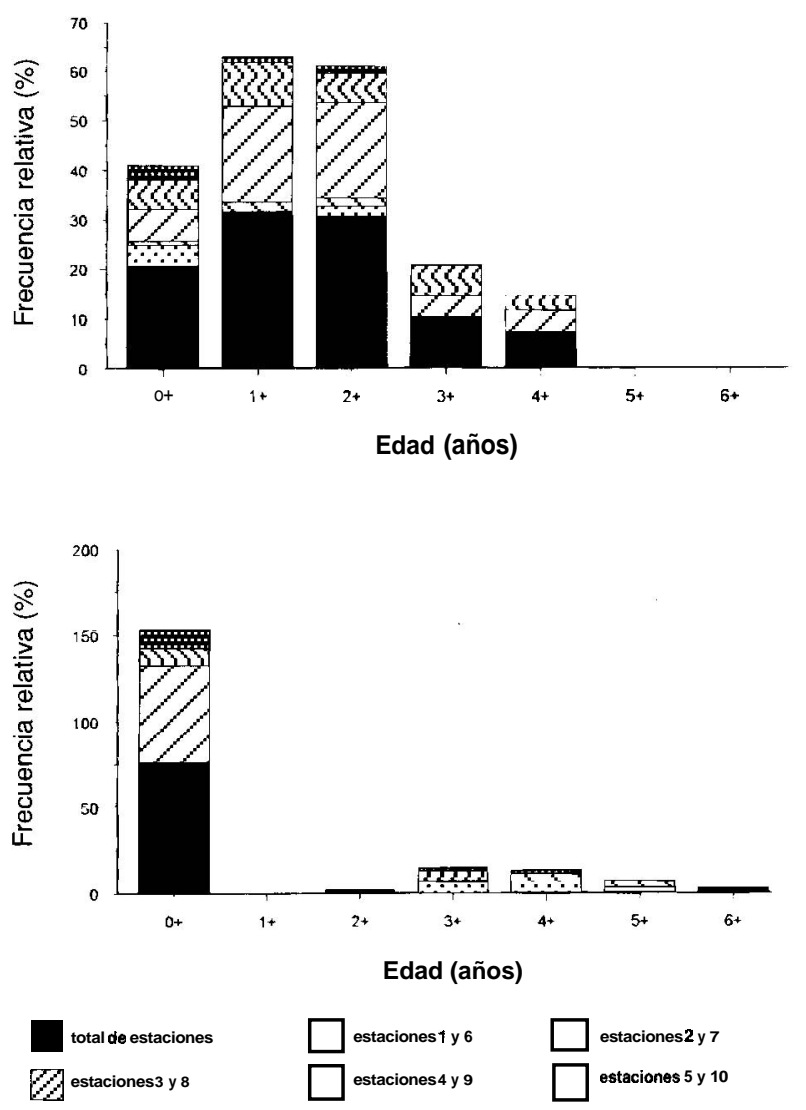

FIGURA 2.- Frecuencia relativa (\%) para las clases de edad del sector A (A) Y B (B).

FIGURE 2.- Relative frecuency (\%) of age classes in site $\mathrm{A}(\mathrm{A})$ and $\mathrm{B}(\mathrm{B})$

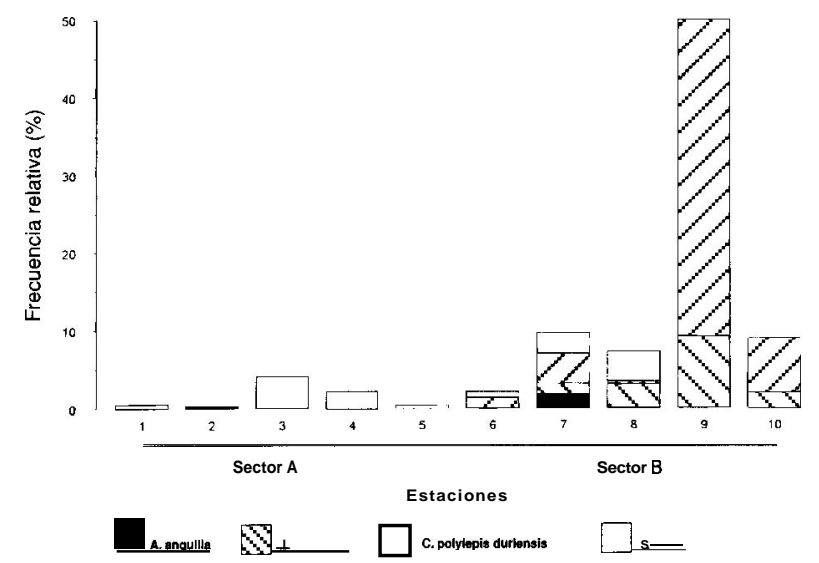

FIGURA 3.- Frecuencia relativa (\%) para las estaciones de muestreo. FIGURE 3.- Relative frecuency (\%) of fish populations for the selected sites
POM), mientras que la tasa específica instantánea de crecimiento sólo se correlaciona con la primera característica.

- El coeficiente para salmónidos está correlacionado positivamente con la cobertura de macrófitos (CM) y su variedad (DSCM) y negativamente con la cantidad de materia orgánica particulada fina (FPOM y DSFPOM) y profundidad media del río (PM). En contrapartida, la producción sólo se correlaciona con la heterogeneidad de la cantidad de cobertura de macrófitos (DSCM).

El factor de condición alométrico se encuentra positivamente influenciado por la cantidad de materia orgánica particulada gruesa (CPOM) y negativamente por el gradiente del canal (GC) (tabla 2). Se verifica que la longitud teórica en el inicio del crecimiento (cuando $t=0$ ) está positivamente influenciada por la anchura media (LM) y heterogeneidad de cobertura de macrófitos (DSCM). La longitud máxima teórica sólo se encuentra influenciada positivamente por la materia orgánica particulada gruesa (CPOM).

Los individuos de S. trutta con peor condición física se encontraron a mayores profundidades, con cantidades más elevadas de materia orgánica particulada fina y gradientes del canal más pronunciados. Hacia las cabeceras, principalmente en zonas con cobertura de macrófitos, se encuentran los individuos con mejor factor de condición, mientras que las mayores tasas instantáneas de crecimiento se obtuvieron en zonas más umbrosas. Hacia los tramos superiores también se encontraron las longitudes máximas teóricas superiores, especialmente en zonas ricas en abrigos, con menor número de alternancias en la relación zonas lénticas-lóticas y menor gradiente del canal al tiempo que presentan mayor cantidad de materia orgánica acumulada.

Los dos ejes de CCA explican $74,9 \%$ de la varianza total, presentando los valores propios $\mathrm{y}_{1}=0,34026 \mathrm{e}$ $\mathrm{y}_{2}=0,14968$ (figura 4). Las correlaciones con estos ejes apenas son significativos para las variables ambientales calidad de zona léntica (QP) $\left(0,718\right.$ con el $2^{\circ}$ eje de las especies y 0,7405 con el $2^{\circ}$ eje de factores ambientales) y desviación standard de la razón zonas lénticas-lóticas (DSPR) (-0,6799 con el 2" eje de los factores ambientales). La correlación obtenida para los ejes especies y factores ambientales, fue bastante alta: 0,9963 para los dos primeros y 0,9696 para los segundos. Los valores del test$\mathrm{t}$ son superiores al valor crítico para $\mathrm{p} \leq 0,05$ para la desviación standard de cobertura de macrófitos (DSCM) en relación al primer eje. 
TABLA 1 - Valores de correlación de Pearson para los datos poblacionales de Salmo trutta en relación a los parámetros ambientales. Leyenda: F', coeficiente para salmónidos: $\mathrm{K}^{\prime}$ factor de condición aiométrico; $\mathrm{P}$, producción; $\mathrm{P} / \overline{\mathrm{B}}$. tasa de renovación: B. biomasa: $\mathrm{L}_{\mathrm{n}} \mathrm{G} \mathrm{g}$, $\mathrm{g}$ y $\mathrm{L}_{\infty}$ son, respectivamente, la longitud teórica para $\mathrm{t}$ $=\mathrm{Q}$ la tasa específica instantánea de crecimiento, la tasa instantánea de decrecimiento de la variable anterior y la longitud máxima teórica del modelo de Von Bertalanffy; LM, anchura media; PM y DSPM, profundidad media y su heterogeneidad; CM y DSCM, \% de cobertura de macrófitos y su heterogeneidad: GC, gradiente del canal; ES, \% de sombra ripícola; FPOM. CPOM, DSFPOM y DSCPOM, \% de materia orgánica particulada fina y gruesa y sus heterogeneidades; SD y DSSD, proporción del sustrato dominante y subdominante y su heterogeneidad; QP, calidad de la zona léntica; PR y DSPR, razón léntica-lótica y su heterogenei$\mathrm{dad} ;{ }^{* *}$, significativo para $\mathrm{p} \leq 0.01 ;{ }^{*}$, significativo para $\mathrm{p} 50.05$.

TABLE 1.- Pearson correlation coefficients between Salmo trutta density and the environmental parameters. Symbols: F'-Condition Factor for salmonids; K' Condition Factor for allometric growth; $\mathrm{P}$-production; $\mathrm{L}_{0} \mathrm{G}_{\mathrm{g}}, \mathrm{g}$ y $\mathrm{L}_{\infty}$ are, respectively, the theoretical length when $t=0$, the size-specific instantaneus rate of growth at $\mathrm{t}=0$, the instantaneus rate of decrease of this rate and the asymptotic length of VBGF model; LM -mean with; PM and DSPM -mean depth and its standard deviation, CM and DSCM -\% macrophyte cover and its standard deviation; GC -canal slope; ES -shadow produced by riparian cover; FPOM, CPOM, DSFPOM and DSCPOM - $\%$ of fine and coarse organic matter and the respective standard deviations; SD and DSSD -dominant and subdominant substrate and its standard deviation; QP -pool quaiity; PR and DSPR - rate of pool/riffle areas and its standard deviation; ${ }^{*}$ values significant for $\mathrm{p} \leq 0.01 ;{ }^{*}$, significant $\mathrm{p} \leq 0.05$.

\begin{tabular}{|c|c|c|c|c|c|c|c|c|}
\hline Parametros & $\mathbf{F}$ & $\mathbf{K}$ & $\mathbf{P}$ & $\mathbf{P} / \overline{\mathbf{B}}$ & $\mathbf{L}_{0}$ & g & G. $\mathrm{g}$ & $\mathbf{L}_{\infty}$ \\
\hline LM & $-0,319$ & $-0,133$ & $-0,045$ & $-0,098$ & $0,847 * *$ & $-0,624$ & $-0,854 * *$ & $0,846^{* *}$ \\
\hline PM & $-0,744 * *$ & 0,116 & 0,022 & $-0,368$ & 0,049 & $-0,580$ & $-0,221$ & 0,546 \\
\hline DSPM & $-0,178$ & 0,310 & $-0,003$ & $-0,317$ & 0,626 & $-0,648$ & $-0,655$ & $0.729 *$ \\
\hline CM & $0,742 * *$ & $-0,076$ & $-0,516$ & 0,039 & 0,208 & 0,120 & $-0,096$ & $-0,100$ \\
\hline DSCM & $0,691 *$ & 0,084 & $-0,664^{*}$ & $-0,123$ & 0,449 & $-0,013$ & $-0,339$ & 0,083 \\
\hline GC & 0,068 & 0,479 & $-0,027$ & 0,466 & 0,056 & $0,767 *$ & 0,295 & $-0,492$ \\
\hline ES & 0,381 & $-0,229$ & $-0,386$ & 0,468 & $-0,410$ & $-0,176$ & 0,198 & 0,081 \\
\hline FPOM & $-0,771 * *$ & 0,557 & 0,248 & $-0,148$ & $-0,016$ & $-0,692$ & $-0,308$ & 0,419 \\
\hline DSFPOM & $-0,744 * *$ & 0,259 & $-0,117$ & $-0,184$ & 0,436 & $-0,845 * *$ & $-0,645$ & $0,822^{*}$ \\
\hline СРОМ & $-0,015$ & $-0,198$ & $-0,354$ & 0,081 & 0,497 & $-0,848 * *$ & $-0,701$ & $0,928 * *$ \\
\hline DSCPOM & $-0,030$ & $-0,099$ & $-0,248$ & $-0,096$ & 0,169 & $-0,719 *$ & $-0,460$ & $0,710^{*}$ \\
\hline SD & 0,148 & 0,427 & $-0,031$ & $-0,199$ & 0,287 & 0,484 & $-0,009$ & $-0,379$ \\
\hline DSSD & $-0,309$ & $-0,181$ & 0,023 & 0,356 & 0,071 & $-0,656$ & $-0,319$ & 0,566 \\
\hline VC & 0,429 & $-0,403$ & $-0,333$ & $-0,123$ & 0,131 & 0,627 & 0,200 & $-0,371$ \\
\hline DSVC & 0,479 & $-0,377$ & $-0,344$ & $-0,071$ & 0,131 & 0,627 & 0,200 & -0371 \\
\hline QP & $-0,464$ & 0,231 & 0,311 & $-0,164$ & $-0,442$ & $-0,041$ & 0,364 & $-0,086$ \\
\hline PR & $-0,156$ & 0,408 & 0,321 & 0,188 & $-0,131$ & $-0,627$ & $-0,200$ & 0,371 \\
\hline DSPR & 0,075 & $-0,259$ & $-0,312$ & 0,073 & 0,131 & 0,627 & 0,200 & $-0,371$ \\
\hline
\end{tabular}

TABLA 2- Regresión múltiple paso a paso para parámetros poblacionales con relación a los parámetros ambientales. Leyenda: $K^{\prime}$, factor de condición alométrico; $\mathbf{L}_{\mathrm{o}}$ y $\mathbf{L}_{\infty}$, son, respectivamente, longitud teórica para $\mathrm{t}=0$ y longitud máxima teórica; $\mathrm{CPOM}$ \% materia orgánica particula gruesa; $\mathrm{GC}$, gradiente del canal; LM. anchura media; DSCM. desviación standard de la cobertura de rnacrófitos; ${ }^{* *}$, significativo para $\mathrm{p} \leq 0,01$; $^{*}$, significativo para $\mathrm{p} \leq 0.05$.

TABLE 2.- Stepwise multiple regression between population and fish parameters. Symbols: $\mathrm{K}^{\prime}$-condition factor for allometric growth; $\mathrm{L}_{0}$ and $\mathrm{L}_{\infty}$ are respectively the theoretical length when $\mathrm{t}=0$ and the asymptotic growth; $\mathrm{CPOM}-\%$ of coarse particulate organic matter; GC -canal slope; LM -mean width; DSCM standard deviation of macrophytic cover; ${ }^{* *}$ correlation coefficients significant for $p \leq 0.01 ;$ * significant for $p \leq 0.05$.

\begin{tabular}{|c|c|c|}
\hline $\mathrm{K}^{\prime}=1,6595+0,0604 \mathrm{CPOM} \cdot \mathbf{0 , 2 1 1 7 \mathrm { GC }}$ & $\operatorname{Radj}=0,897 * *$ & $(\mathrm{GL}=5)$ \\
\hline $\mathrm{L}_{0}=32,501044+2,449009 \mathrm{LM}+0,25795 \mathrm{DSCM}$ & $\operatorname{Radj}=0,959^{* *}$ & $(\mathrm{GL}=5)$ \\
\hline $\mathrm{L}_{\infty}=23,9864+2,064 \mathrm{CPOM}$ & $\operatorname{Radj}=0,826^{*}$ & $(\mathrm{GL}=5)$ \\
\hline
\end{tabular}

En la figura 4 se evidencia que las características bióticas y abióticas de los sectores A y B son claramente distintas. Además, se observa que la desviación standard de cobertura de macrófitos es la variable ambiental más significativa en el proceso de ordenación. Esta variable condiciona positivamente las densidades encontradas en la totalidad de especies y en el caso de S. trutta, especialmente a los individuos de edad inferior $(0+$ y $1+)$, posiblemente por la mayor cantidad de alimento encontrado así como por la posibilidad de refugio para estos individuos más jóvenes. Esta variable condiciona negativamente a Leuciscus cephalus, y a que esta especie se encuentra localizada más hacia abajo, en zonas con menor presencia de cobertura de macrófitos (CM).

La calidad de la zona Iéntica condiciona positivamente los individuos de clases de edad superiores de S. trutta 

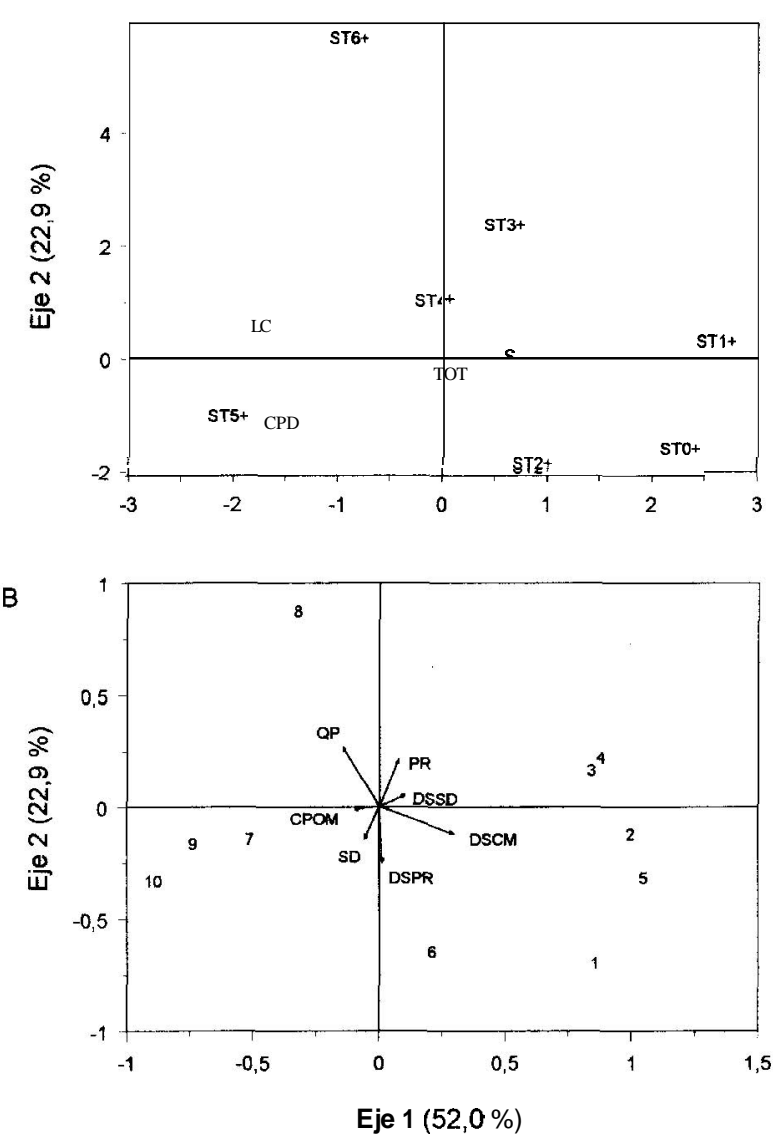

FIGURA 4.- Análisis de los valores de densidad (A) y de los parámetros ambientales (B) a partir de CCA. Leyenda: tot, densidad total de peces; ST, CPD y LC, son, respectivamente, la densidad total de S. trutta, C. polylepis duriensis y L. cephalus; ST1+, ST2+, ST3+, ST4+, ST5 y ST6+, son las densidades de S. trutta para las edades $\mathrm{O}+1+, 2+, 3+, 4+, 5+$ y $6+$, respectivamente; QP, calidad de zona léntica; PR y DSPR, razón Iéntica-lótica y su heterogeneidad; SD y DSSD, sustrato y su heterogeneidad; DSCM, heterogeneidad de cobertura de macrófitos; CPOM, cantidad de materia orgánica particulada gruesa.

FIGURE 4.- CCA ordination of fish densities (A) and of the environmental parameters (B). Symbols: tot -overal fish density; ST, CPD and LC are, respectively, total abundances of S. trutta, C. polylepis duriensis and $L$. cephalus; ST1+, ST2+, ST3+, ST4+, ST5+ and ST6+ are S. trutta densities for age classes $\mathrm{O}+1+, 2+, 3+, 4+, 5+$ and $6+; \mathrm{QP}$-quality of pool; $\mathrm{RR}$ and DSPR are the riffle/pool rate and its standard deviation; SD and DSSD are the dominant substrate and its standard deviation; DSCM -standard deviation of macrophytic cover; CPOM -coarse particulate organic matter.

(4+ y 6+) al permitir muy probablemente una relación entre zonas de refugio y alimento, e influencia negativamente las densidades de los individuos de tamaño intermedio (2+) así como la densidad total piscícola. A medida que aumenta la razón entre zonas lénticas-lóticas, aumenta la proporción relativa de la clase $3+$.
Tanto la razón zona léntica-lótica como su heterogeneidad determinaron las bajas densidades de individuos de S. trutta, de clases 4+ y 5+, y de Chondrostoma polylepis duriensis.

\section{DISCUSION}

El método de muestreo adoptado (pesca eléctrica) ha sido usado por numerosos autores como por ej. EGGLISHAW \& SHACKLEY (1982) para estudiar el uso particular del hábitat por individuos de trucha. Este procedimiento apenas nos conduce a una estima muy grosera del uso del hábitat, esto es, número y tamaño de individuos observados en una determinada sección del río relativamente grande (HEGGENES, 1988b), no siendo posible hacer un análisis más pormenorizado.

En relación a las variables del hábitat, se puede considerar que han sido más reiteradamente estudiadas cuatro variables físicas del hábitat: profundidad y velocidad (o gradiente del lecho) del río, sustrato y cobertura, consideradas, tal como HEGGENES (1988b), como las más importantes. Muchas de las características consideradas por PLATTS $\boldsymbol{e}$ t al. (1983, 1987) son, en general, variaciones refinadas de las consideradas anteriormente. Sin embargo, en el río Olo debido al hecho de presentar una baja productividad primaria y una gran homogeneidad en la velocidad de corriente y profundidad del lecho, se reveló que los factores ambientales más importantes eran la cobertura de macrófitos y acumulación de materia orgánica, además del tipo de sustrato. Estos factores crean una mayor heterogeneidad del hábitat al ofrecer zonas específicas para cada una de las clases de edad de S. trutta y especies asociadas.

S. trutta se caracteriza por una fuerte competición intraespecífica, siendo el factor "tamaño del individuo" el que impone la jerarquía, relegando a los individuos más pequeños a los hábitats subóptimos (HEGGENES, 1988a), al igual que otras especies (NELISSEN, 1992). De esta forma, se hace necesario considerar la composición total de la población y no sólo un reducido número de clases de edad.

Diferentes estudios consideran distintamente la importancia de las variables del hábitat, que conduce a una aparente confusión en considerar aquella variable del hábitat más relevante, en el caso de existir alguna. Numerosos estudios con S. trutta indican que la profundidad del agua es una característica física importante que condiciona la bondad del hábitat. EGGLISHAW \& SHAGKLEY (1982) en- 
contraron una correlación positiva entre las densidades de poblaciones de $S$. trutta de O+ y $1+$ simpátricas con poblaciones de Salmo salar, en áreas con una profundidad superior a $10 \mathrm{~cm}$, siendo esta correlación más fuerte para los individuos I+. Resultados similares fueron encontrados por KENNEDY \& STRANGE $(1982,1986)$ con preferencias significativas de los alevines de ambas especies por hábitats poco profundos $(<20 \mathrm{~cm})$ y de los individuos más adultos de $S$. trutta $(\geq 2+)$ por aguas más profundas $(>20$ $\mathrm{cm})$.

La importancia simultánea de la profundidad y velocidad de la corriente fue evidenciada en poblaciones simpátricas de $S$. trutta y $S$. salar por BAGLINIÈRE \& GHAMPIGNEULLE (1982). Los individuos del año se encontraban en zonas de rápidos poco profundos (10-40 $\mathrm{cm})$, mientras que los individuos de más edad colonizaban todo tipo de hábitats siempre que les garantizasen cierta seguridad, tal como la profundidad y cobertura. GATZ et al. (1987) encontraron también que la profundidad del agua apenas es importante para separar el uso del hábitat por edades O+ y >O+ de S. trutta. KENNEDY \& STRANGE (1982) y GUNJAK \& POWER (1986) refieren que los individuos de trucha aumentan el uso de habitats más profundos y con sustrato más grosero, a medida que el tamaño del pez aumenta desde O+ a edades superiores.

La función principal del sustrato del río como hábitat es, aparentemente, ofrecer un refugio frente a los depredadores o fuertes velocidades de corriente. Así mismo, el sustrato más grueso es ventajoso desde el punto de vista de minimizar la energía, teniendo en cuenta las bajas velocidades de agua encontradas en los nichos creados en los espacios intersticiales entre partículas (HEGGENES, 1988b).

Atendiendo a un punto de vista energético, S. trutta debe ocupar en aguas corrientes microhábitats que suministren localmente bajas velocidades de agua (FAUSCH, 1984). CUNJAK \& POWER (1986) encontraron en la estación invernal para los individuos de edad O+ bajas velocidades locales con valores medios de 2,2-4,7 cm s-', determinándose para los individuos más adultos velocidades de $5,7-16,0 \mathrm{~cm} \mathrm{~s}^{-1}$. Existen por eso escasas dudas sobre la selección de microhábitats de baja velocidad del agua por esta especie, que llevan a una minimización del gasto energético.

Donde el río se ensancha, este tipo de individuos se asocia frecuentemente con las márgenes o con zonas que presentan ángulos de las márgenes invertidos, en donde la velocidad del agua es moderada y la densidad de indivi- duos adultos menor (BAGLINIÈRE \& CHAMPIGNEU. LLE, 1982; observación personal). Por otro lado existe una tendencia aparente para el surgimiento de una segregación competitiva entre individuos 0+ y 1+ (BAGLINIÈ RE \& CHAMPIGNEULLE, 1982; EGGLISHAW \& SHACKLEY, 1982; KENNEDY \& STRANGE, 1982; CUNJAK \& POWER, 1986; GATZ et al., 1987).

En este trabajo se optó por la descripción general de la velocidad del agua en los locales preferidos por S. trutta y no por la cuantificación de la velocidad microfocal, ya que desde el punto de vista de ordenación del territorio este abordaje es más útil. La importancia en definir velocidades del agua apropiadas para $S$. trutta ha sido enfatizado por un gran número de autores. Así, las truchas de menor tamaño son encontradas por lo general en zonas lóticas de poca profundidad y velocidad del agua moderada (20-50 $\mathrm{cm} \mathrm{s}^{-1}$ ) (HEGGENES, 1988b).

Los estudios anteriormente referidos, al igual que éste enfocan el uso del hábitat de verano. Según los datos de CUNJAK \& POWER (1986) la distribución de los individuos en el invierno es consistente con los datos observados en el verano tanto para $S$. trutta como para Salvelinus fontinalis, ocupando los individuos más adultos $(>1+)$ frente a los individuos O+ las zonas más profundas.

\section{BIBLIOGRAFÍA}

BAGLINIÈRE, J.-L. \& CHAMPIGNEULLE, A., 1982. Densité des populations de truite commune (Salmo trutta L.) et de juvéniles de saumon atlantique (Salmo salar L.) sur le cours principal du Scorff (Bretagne): préférendums physiques et variations annuelles (1976-1980). Acta Ecologica Ecol. Applic., 3 (3): 241-256.

CUNJAK, R. A. \& POWER, G., 1986. Winter habitat utilization by stream resident brook trout (Salvelinus fontinalis) and brown trout (Salmo trutta). Can. J. Fish. Aquat. Sci., 43: 1970-1981.

EGGLISHAW, H. J. \& SHACKLEY, P. E., 1982. Influence of water depth on dispersion of juvenile salmonids Salmo salar L. and Salmo trutta L. in a Scottish stream. J. Fish. Biol., 21: 141-155.

ELLIOTT, J. M., 1989. Wild brown trout Salmo trutta: an important national and international resource. Fresh water Biol., 21: 1-5.

FAUSCH, K. D., 1984. Profitable stream positions for salmonids: relating specific growth rate to net energy gain. Can. J.Zool., 62: 441-451.

GATZ, A. J., Jr., SALE, M. J. \& LOAR, J. M., 1987. 
Habitat shifts in rainbow trout: cornpetitive influences of brown trout. Ecologia, 74: 7-19.

HEGGENES, J., 1988a. Effect of experimentally increased intraspecific competition on sedentary adult brown trout (Salmo trutta) rnovernent and strearn habitat choice. Can. J. Fish. Aquat. Sci., 45:1163-1172.

HEGGENES, J., 1988b. Physical habitat selection by brown trout (Salmo trutta) in riverine systerns. Nordic. J. Freshw. Res., 64: 74-90.

KENNEDY, G. J. A. \& STRAnGE, C. D., 1982. The distribution of salrnonids in upland strearns in relation to depth and gradient. J. Fish. Biol., 20: 579-591.

KENNEDY, G. J. A. \& STRANGE, C. D., 1986. The effects of intra and inter-specific cornpetition on the distribution of stocked juvenile Atlantic salmon, Salmo salar, in relation to depth and gradient in an upland trout, Salmo trutta L., stream. J. Fish Biol., 29: 199-214.

NELISSEN, M. H. J., 1992. Does body size affect the ranking of a ciclhild fish in a dorninance hierarchy?. J. Ethol., 10:153-156.

PLATTS, W. S., MEGAHAN, W. F. \& MINSHALL, G. W., 1983. Methods for evaluation strearn, riparian, and biotic conditions. United States Department of Agriculture, Forest Service, Interrnountain Forest and Range Experirnent Station Ogden, General Technical Report INT, 138: $1-70$.
PlATTS, W. S., ARMOUR, C., BOOTH, G. D., BRYANT, M., BUFFORD, J. L., CUPLIN, P., JENSEN, S., LIENKAEMPER, G. W., MINSHALL, G. W., MONSEN, S. B., NELSON, R. L., SEDELL, J. R. \& TUHY, J. S., 1987. Methods for evaluating riparian habitats with applications to management. United States Department of Agriculture, Forest Service, Interrnountain Research Station, General Technical Report INT, 221:1-177.

SOKAL, R. R. \& ROHLF, F. J., 1969. Biometry. The principles and practice of statistics in biological research. Ed. W. H. Freernan and Cornpany, San Francisco. 776 p.

TER BRAAK, J. F., 1987. CANOCO - a fortran program for canonical community ordination by correspondence analysis, principal components analysis and redundancy analysis. Ed. TNO Institute of Applied Computer Science. 82 p. 